Supporting Information:

\title{
Rational Design and First Principles Studies Toward the Remote Substituent Effects on a Novel Tetracyclic Proton Sponge
}

Ajeet Singh and Bishwajit Ganguly*

Table of Content:

B3LYP/6-311+G**//B3LYP/6-31G* SCF energies, .........................Page S2-S11

B3LYP/6-31G* zero point vibrational energies (ZPVE),

Cartesian coordinates for 1-7 and their monoprotonated ions

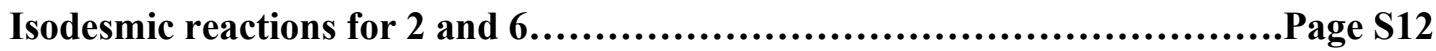




\section{B3LYP/6-311+G**//B3LYP/6-31G* SCF energies, B3LYP/6-31G* zero point vibrational energies (ZPVE), and Cartesian coordinates for 1-7 and their monoprotonated ions}

\begin{tabular}{|c|c|c|c|}
\hline \multicolumn{4}{|c|}{$\begin{aligned} \mathrm{SCF} \text { ENERGY at B3LYP } / 6-311+\mathrm{G}^{* *} & =-578.92272 \mathrm{au} \\
\mathrm{ZPVE} & =201.265 \mathrm{kcal} /\end{aligned}$} \\
\hline atom & $\mathrm{x}$ & $\mathrm{y}$ & $\mathrm{z}$ \\
\hline $\mathrm{C} 1$ & 0.1228361005 & -3.8255106670 & 1.6966925203 \\
\hline $\mathrm{C} 2$ & 0.3210373441 & -3.1855838522 & 3.0910819439 \\
\hline $\mathrm{C} 3$ & 0.3637251137 & -1.6363812596 & 2.8758189806 \\
\hline $\mathrm{C} 4$ & 2.3074051597 & -2.9179559801 & 2.2063565673 \\
\hline $\mathrm{C} 5$ & -0.0606465569 & -5.3572587750 & 1.5758710371 \\
\hline C6 & -0.3464037829 & -5.6615838525 & 0.0679300898 \\
\hline $\mathrm{C} 7$ & 1.0678913637 & -5.4698223667 & -0.5648983710 \\
\hline $\mathrm{C} 8$ & 1.9239435596 & -5.0883272441 & 0.6882621125 \\
\hline H9 & -0.4438761297 & -1.2788217953 & 2.2283767542 \\
\hline $\mathrm{H} 10$ & -0.7794718124 & -5.7935627986 & 2.2762902725 \\
\hline H11 & -0.7095394110 & -6.6863813060 & -0.0620520253 \\
\hline H12 & -1.1016287192 & -4.9943740535 & -0.3605535112 \\
\hline H13 & 1.4330594801 & -6.3953831966 & -1.0219994254 \\
\hline H14 & 1.0888287132 & -4.6966197140 & -1.3401100220 \\
\hline $\mathrm{C} 15$ & 1.7803830332 & -1.4452260952 & 2.2480659372 \\
\hline N16 & 1.7166724710 & -3.5409070046 & 3.4036691173 \\
\hline N17 & 1.3100041501 & -5.8553309048 & 1.7861007970 \\
\hline H18 & 2.9962420922 & -5.2822134401 & 0.5877383026 \\
\hline H19 & 0.2717451590 & -1.1064702444 & 3.8295298277 \\
\hline $\mathrm{C} 20$ & 1.4968053889 & -7.2953248450 & 1.7662283041 \\
\hline $\mathrm{C} 21$ & 2.2322901858 & -3.1083230802 & 4.6906909127 \\
\hline $\mathrm{H} 22$ & 2.5622048652 & -7.5171037529 & 1.8982841897 \\
\hline $\mathrm{H} 23$ & 0.9610779393 & -7.7344046436 & 2.6158891872 \\
\hline $\mathrm{H} 24$ & 1.1574929442 & -7.8161748978 & 0.8513178754 \\
\hline $\mathrm{H} 25$ & 1.6812929338 & -3.6246561877 & 5.4853668964 \\
\hline $\mathrm{H} 26$ & 3.2837431363 & -3.4078955176 & 4.7705727942 \\
\hline H27 & 2.1769541277 & -2.0229144547 & 4.8958170914 \\
\hline $\mathrm{H} 28$ & -0.3818131579 & -3.5227527630 & 3.8593561522 \\
\hline $\mathrm{H} 29$ & 2.4189680344 & -0.8179903945 & 2.8788332536 \\
\hline $\mathrm{C} 30$ & 1.5543049724 & -3.6321357260 & 1.0580344833 \\
\hline H31 & 3.3970199645 & -3.0149485522 & 2.1754892888 \\
\hline H32 & 1.7502744038 & -0.9813625159 & 1.2566196026 \\
\hline ? & -0.6749684869 & -3.3082674849 & 1.1546432623 \\
\hline & 1.5543837266 & -3.0047355502 & 0.1614547 \\
\hline
\end{tabular}

\section{$1 \mathbf{H}^{+}$}

SCF ENERGY at B3LYP/6-311+G** = -579.34449au $\mathrm{ZPVE}=209.581 \mathrm{kcal} / \mathrm{mol}$

$\begin{array}{lccc}\text { atom } & \mathrm{x} & \mathrm{y} & \mathrm{z} \\ \text { C1 } & 0.0850434866 & -3.7696965542 & 1.6379251314 \\ \text { C2 } & 0.2934943815 & -3.1818169662 & 3.0554128413 \\ \text { C3 } & 0.3907282322 & -1.6388782243 & 2.9333547171 \\ \text { C4 } & 2.2987375556 & -2.9120819846 & 2.1623409166 \\ \text { C5 } & -0.1159933305 & -5.2987474824 & 1.5516993105 \\ \text { C6 } & -0.3304301614 & -5.7177248809 & 0.0845366036 \\ \text { C7 } & 1.0875884408 & -5.5287230876 & -0.5453988748 \\ \text { C8 } & 1.9293800387 & -5.0254756842 & 0.6428300279 \\ \text { H9 } & -0.4133404014 & -1.2287703307 & 2.3157550545 \\ \text { H10 } & -0.8108288099 & -5.7245746262 & 2.2784325507 \\ \text { H11 } & -0.6758930232 & -6.7522016259 & 0.0063445466\end{array}$




$\begin{array}{lrrr}\text { H12 } & -1.0913895016 & -5.0903556099 & -0.3855267652 \\ \text { H13 } & 1.4809819205 & -6.4647618238 & -0.9512093868 \\ \text { H14 } & 1.0975929048 & -4.7989250672 & -1.3583993008 \\ \text { C15 } & 1.8048119677 & -1.4486241961 & 2.3025676795 \\ \text { N16 } & 1.6961030263 & -3.6263362979 & 3.3317398253 \\ \text { N17 } & 1.3095839964 & -5.7779563882 & 1.8190524727 \\ \text { H18 } & 3.0031595991 & -5.2147307299 & 0.5840320812 \\ \text { H19 } & 0.3157242609 & -1.1626706913 & 3.9144548893 \\ \text { C20 } & 1.5539200466 & -7.2377020349 & 1.9299403755 \\ \text { C21 } & 2.2408559137 & -3.2939936530 & 4.6546967309 \\ \text { H22 } & 2.6237046125 & -7.4030439664 & 2.0748314098 \\ \text { H23 } & 1.0077689529 & -7.6186110077 & 2.7956214067 \\ \text { H24 } & 1.2224838713 & -7.7579416985 & 1.0314260116 \\ \text { H25 } & 1.6767762725 & -3.8384404215 & 5.4175352825 \\ \text { H26 } & 3.2832623498 & -3.6222134702 & 4.7018590164 \\ \text { H27 } & 2.2038641387 & -2.2240143874 & 4.8949279713 \\ \text { H28 } & -0.4112025247 & -3.5449355922 & 3.8081842229 \\ \text { H29 } & 2.4665319580 & -0.8725525982 & 2.9546411370 \\ \text { C30 } & 1.5254997958 & -3.5770862460 & 0.9970060226 \\ \text { H31 } & 3.3842049506 & -3.0339921335 & 2.1179567974 \\ \text { H32 } & 1.7785627855 & -0.9343219354 & 1.3377105784 \\ \text { H33 } & -0.7156095142 & -3.2555861512 & 1.1027591607 \\ \text { H34 } & 1.5368178713 & -2.9549603955 & 0.1000253932 \\ \text { H35 } & 1.6255249241 & -5.2475730015 & 2.6899013208\end{array}$

2

SCF ENERGY at B3LYP/6-311+G** = -657.54966 au

\begin{tabular}{lccc} 
& & \multicolumn{2}{c}{ ZPVE $=238.424 \mathrm{kcal} / \mathrm{mol}$} \\
atom & $\mathrm{x}$ & $\mathrm{y}$ & $\mathrm{z}$ \\
$\mathrm{C} 1$ & 0.0551697057 & -3.7197318918 & 1.6683494835 \\
$\mathrm{C} 2$ & 0.4589273728 & -3.1559053153 & 3.0808048577 \\
$\mathrm{C} 3$ & 0.5713636744 & -1.5961400800 & 3.0407251594 \\
$\mathrm{C} 5$ & 2.3297474716 & -2.8886797093 & 1.9843961414 \\
C6 & -0.1125386743 & -5.2811806916 & 1.6491787159 \\
C7 & -0.4685331919 & -5.7948326608 & 0.2113287790 \\
C8 & 0.9026482384 & -5.6597391757 & -0.5190124257 \\
C9 & 1.8006368310 & -5.0666595201 & 0.6163872592 \\
H10 & -0.2837482178 & -1.0809014757 & 2.5999840456 \\
H11 & -0.7770218553 & -5.6587353728 & 2.4337040022 \\
H12 & -0.7847763925 & -6.8418009934 & 0.2708922931 \\
H13 & -1.2785541396 & -5.2585901158 & -0.2865681182 \\
H14 & 1.2929941528 & -6.6399010795 & -0.8125184263 \\
H15 & 0.8545402105 & -5.0625757803 & -1.4309314437 \\
C16 & 1.8960681795 & -1.4036246251 & 2.2407246128 \\
N17 & 1.8669834805 & -3.5639086678 & 3.2037986162 \\
N18 & 1.2727497455 & -5.7420210840 & 1.8117154589 \\
H19 & 2.8730687151 & -5.2382400660 & 0.4752238341 \\
H20 & 0.6672199927 & -1.2149820631 & 4.0630995645 \\
C23 & 1.5045860007 & -7.1666737418 & 1.9421371476 \\
C24 & 2.5526599708 & -3.2687570483 & 4.4458727095 \\
H25 & 2.5835130463 & -7.3420131135 & 2.0284327673 \\
H26 & 1.0394073822 & -7.5167417745 & 2.8712873003 \\
H27 & 1.1285477100 & -7.8063865306 & 1.1209333798 \\
H28 & 2.0745834637 & -3.8326032397 & 5.2556466834 \\
H29 & 3.5888298297 & -3.6190680621 & 4.3687221366 \\
H30 & 2.5834856540 & -2.2060552983 & 4.7537734038 \\
H31 & -0.1629580100 & -3.5396640807 & 3.8965157347 \\
H32 & 2.6584058501 & -0.9059449586 & 2.8497406898 \\
C33 & 1.4380727703 & -3.5583828536 & 0.8788645527 \\
H34 & 3.4043670380 & -3.0150295279 & 1.8146655551 \\
H35 & 1.7746702253 & -0.7931500678 & 1.3439915403 \\
C36 & 1.4226955779 & -2.7471162645 & -0.4241050255
\end{tabular}




$\begin{array}{lrrr}\text { C38 } & -1.1751787932 & -2.9976555676 & 1.1010276006 \\ \text { H39 } & -1.4788750269 & -3.3869588864 & 0.1277228502 \\ \text { H40 } & -2.0319395533 & -3.1113125775 & 1.7787202296 \\ \text { H41 } & -1.0069365576 & -1.9272720290 & 0.9657688942 \\ \text { H42 } & 1.2021629362 & -1.6915181903 & -0.2584510120 \\ \text { H43 } & 2.4008951798 & -2.8013318812 & -0.9203430721 \\ \text { H44 } & 0.6767187919 & -3.1102027195 & -1.1337340735\end{array}$

\section{$\mathbf{2 H}^{+}$}

SCF ENERGY at B3LYP/6-311+G** $=-657.98049 \mathrm{au}$ $\mathrm{ZPVE}=246.800 \mathrm{kcal} / \mathrm{mol}$

\begin{tabular}{|c|c|c|c|}
\hline NII & X & & \\
\hline $\mathrm{C} 1$ & 0.0336139150 & -3.6979191205 & 1.5490926512 \\
\hline $\mathrm{C} 2$ & 0.2758665111 & -3.1574476940 & 3.0062336210 \\
\hline $\mathrm{C} 3$ & 0.4466408467 & -1.6158400960 & 3.0234240770 \\
\hline $\mathrm{C} 4$ & 2.2862659851 & -2.9019973681 & 2.1222226790 \\
\hline $\mathrm{C} 5$ & -0.1263471048 & -5.2589290005 & 1.5264419826 \\
\hline C6 & -0.3066925386 & -5.8212500922 & 0.0999002188 \\
\hline $\mathrm{C} 7$ & 1.1127732756 & -5.6402352875 & -0.5246619067 \\
\hline $\mathrm{C} 8$ & 1.9140751678 & -4.9986773579 & 0.6284523434 \\
\hline H9 & -0.3358519082 & -1.0825991959 & 2.4835945964 \\
\hline $\mathrm{H} 10$ & -0.8242376911 & -5.6489746856 & 2.2716241763 \\
\hline H11 & -0.5987784221 & -6.8745189276 & 0.1390168457 \\
\hline H12 & -1.0910200090 & -5.3079113604 & -0.4544128041 \\
\hline H13 & 1.5541803387 & -6.5999807756 & -0.8080333536 \\
\hline H14 & 1.1136 & -5.0267499955 & -1.4244602221 \\
\hline $\mathrm{C} 15$ & 1.8649 & -1.4355527121 & 2.4016128901 \\
\hline N16 & 1.6690 & -3.6 & 618930 \\
\hline N17 & 1.2980000525 & -5.7025147749 & 1.8294471963 \\
\hline H18 & 2.99407 & -5.16208 & 0.5914240131 \\
\hline H19 & 0.4102 & -1.2 & 38971034 \\
\hline $\mathrm{C} 20$ & 1.568 & -7.14690 & 1979474 \\
\hline $\mathrm{C} 21$ & 2.226 & -3.4014 & +768933 \\
\hline $\mathrm{H} 22$ & 2.6407467380 & -7.2799167246 & 2.1875596666 \\
\hline $\mathrm{H} 23$ & 1.0260542467 & -7.4844943278 & 2.9111942114 \\
\hline $\mathrm{H} 24$ & 1.2572 & -7.7 & 8437194 \\
\hline $\mathrm{H} 25$ & 1.6452053669 & -3.97088 & 5815276 \\
\hline $\mathrm{H} 26$ & 3.2576026622 & -3.7661680118 & 4.6300454483 \\
\hline $\mathrm{H} 27$ & 2.22594 & -2.3482 & 4.9105484219 \\
\hline $\mathrm{H} 28$ & -0.4389383612 & -3.5430050818 & 3.7393166314 \\
\hline $\mathrm{H} 29$ & 2.56035 & -0.9 & 0043981 \\
\hline $\mathrm{C} 30$ & 1.4972240094 & -3.51156 & 1274192 \\
\hline H31 & 3.3672167730 & -3.0608423430 & 2.0646855418 \\
\hline H32 & & & 79470 \\
\hline $\mathrm{C} 33$ & 1.6139924295 & -2.6554718731 & -0.3606631130 \\
\hline C34 & -1.1448873090 & -3.0070024281 & 0.8539380873 \\
\hline H35 & -1.2765615529 & -3.3395466285 & -0.1772780853 \\
\hline H36 & -2.0801162360 & -3.2099631414 & 1.3894514834 \\
\hline H37 & -1.0189837205 & -1.9244209711 & 0.8151712378 \\
\hline H38 & 1.2797091744 & -1.6309648682 & -0.1932696259 \\
\hline H39 & 2.6557396380 & -2.6102123678 & -0.7004256360 \\
\hline $\mathrm{H} 40$ & 1.0146619240 & -3.0447541224 & -1.1856455212 \\
\hline H41 & 1.5988787323 & -5.1026034336 & 2.6868579329 \\
\hline
\end{tabular}


3

SCF ENERGY at B3LYP/6-311+G** $=-736.19363 \mathrm{au}$ $\mathrm{ZPVE}=274.951 \mathrm{kcal} / \mathrm{mol}$

\begin{tabular}{|c|c|c|c|}
\hline III & $X$ & & \\
\hline $\mathrm{C} 1$ & 0.0274116713 & -3.7150917901 & 1.6278089389 \\
\hline $\mathrm{C} 2$ & 0.4074784487 & -3.0453509412 & 3.0005370156 \\
\hline $\mathrm{C} 3$ & 0.5104977581 & -1.4889034682 & 2.8514510864 \\
\hline $\mathrm{C} 4$ & 2.2930850543 & -2.8339511645 & 1.9139873880 \\
\hline C5 & -0.0865301093 & -5.2823435505 & 1.7125980327 \\
\hline C6 & -0.4552021510 & -5.8914692946 & 0.3165844370 \\
\hline $\mathrm{C} 7$ & 0.8933701316 & -5.7456736230 & -0.4542457062 \\
\hline $\mathrm{C} 8$ & 1.8023963631 & -5.0746387278 & 0.6313857162 \\
\hline $\mathrm{H} 9$ & -0.3348654298 & -1.0131354076 & 2.3506138895 \\
\hline H10 & -0.7177087216 & -5.6360278928 & 2.5340377726 \\
\hline H11 & -0.7267089573 & -6.9450052449 & 0.4434760627 \\
\hline H12 & -1.2975175340 & -5.4163056760 & -0.1899670090 \\
\hline H13 & 1.3074213764 & -6.7252437840 & -0.7162573538 \\
\hline H14 & 0.7999448286 & -5.1908615934 & -1.3898344428 \\
\hline $\mathrm{C} 15$ & 1.8556 & -1.33781 & 2.0756151696 \\
\hline N16 & 1.8140 & -3.4351 & 57679015 \\
\hline N17 & 1.31454 & -5.6936 & 1.8711673698 \\
\hline H18 & 2.8738 & -5.2392 & 0.4800552755 \\
\hline H19 & 0.5744 & $-1.034 \mathrm{C}$ & 3.8462407232 \\
\hline $\mathrm{C} 20$ & 1.5874 & -7.1017 & 2.0790093226 \\
\hline $\mathrm{C} 21$ & 2.4802 & -3.059763 & 70361196 \\
\hline $\mathrm{H} 22$ & 2.671 & -7.2 & 88042703 \\
\hline $\mathrm{H} 23$ & 1.144 & -7.410 & 5985977 \\
\hline $\mathrm{H} 24$ & 1.2176333175 & -7.797203 & 1.3013665258 \\
\hline $\mathrm{H} 25$ & 1.993 & -3.5 & 3201709 \\
\hline $\mathrm{H} 26$ & 3.5193 & -3.4076 & 4.3569796263 \\
\hline $\mathrm{H} 27$ & & & \\
\hline $\mathrm{H} 28$ & -0.214 & -3.3 & 7352688 \\
\hline $\mathrm{H} 29$ & 2.6015402901 & -0.80675 & 2.6766847271 \\
\hline C30 & 1.4136 & -3.562 & 0.8303674368 \\
\hline H31 & 3.3700346632 & -2.9709936893 & 1.7741269699 \\
\hline H32 & 1.7575388913 & -0.7788376111 & 1.1429702413 \\
\hline $\mathrm{C} 33$ & 1.3661 & -2.7887306009 & -0.5040018998 \\
\hline C34 & -1.2207547748 & -3.0713158561 & 0.9880330817 \\
\hline $\mathrm{C} 35$ & 2.7218235222 & -2.6842163952 & -1.2181769435 \\
\hline $\mathrm{C} 36$ & -2.5082 & -3.2533522330 & 1.8058706291 \\
\hline H37 & 2.6231687607 & -2.1257666992 & -2.1564306131 \\
\hline H38 & 3.46 & -2.1654790060 & 27853031 \\
\hline H39 & 3.1281237901 & -3.6720883559 & -1.4643360813 \\
\hline $\mathrm{H} 40$ & -3.3568190763 & -2.7740394923 & 1.3041062737 \\
\hline H41 & -2.7589119731 & -4.3122522746 & 1.9381037466 \\
\hline $\mathrm{H} 42$ & -2.4215517412 & -2.8078183493 & 2.8036216580 \\
\hline H43 & 0.6464565755 & -3.2539708177 & -1.1835141452 \\
\hline H44 & 0.9776701767 & -1.7796406837 & -0.3400163403 \\
\hline $\mathrm{H} 45$ & -1.0538325539 & -2.0021673833 & 0.8296828862 \\
\hline H46 & -1.3813256811 & -3.4762520473 & -0.0151569681 \\
\hline
\end{tabular}

$3 \mathbf{H}^{+}$

SCF ENERGY at B3LYP/6-311+G** = -736.62598 au $\mathrm{ZPVE}=281.775 \mathrm{kcal} / \mathrm{mol}$

$\begin{array}{lccc}\text { atom } & \mathrm{x} & \mathrm{y} & \mathrm{z} \\ \mathrm{C} 1 & 0.0048030468 & -3.6894184922 & 1.6039523647 \\ \text { C2 } & 0.3847948026 & -3.0435177188 & 2.9881955471 \\ \text { C3 } & 0.5343234821 & -1.5029748223 & 2.8763757517\end{array}$




\begin{tabular}{|c|c|c|c|}
\hline $\mathrm{C} 4$ & 2.2955511221 & -2.8384765516 & 1.8931248726 \\
\hline $\mathrm{C} 5$ & -0.1300654660 & -5.2503963174 & 1.7078901702 \\
\hline C6 & -0.4426563031 & -5.9127057896 & 0.3483476434 \\
\hline $\mathrm{C} 7$ & 0.9050504128 & -5.7657653184 & -0.4267038321 \\
\hline $\mathrm{C} 8$ & 1.8081203315 & -5.0398333393 & 0.5944010770 \\
\hline H9 & -0.3039894102 & -1.0168518152 & 2.3769776244 \\
\hline $\mathrm{H} 10$ & -0.7385452961 & -5.5983488982 & 2.5452850901 \\
\hline H11 & -0.7145619601 & -6.9628698902 & 0.4883489332 \\
\hline H12 & -1.2840178991 & -5.4432585802 & -0.1596331998 \\
\hline H13 & 1.3282210101 & -6.7399602498 & -0.6877850511 \\
\hline H14 & 0.8054997535 & -5.2138341837 & -1.3604197124 \\
\hline $\mathrm{C} 15$ & 1.8822570749 & -1.3582758613 & 2.1061368131 \\
\hline N16 & 1.8028387192 & -3.4984464577 & 3.1440198619 \\
\hline N17 & 1.3226671773 & -5.6581400739 & 1.8964073353 \\
\hline H18 & 2.8799928151 & -5.2050164499 & 0.4668652912 \\
\hline H19 & 0.5937751021 & -1.0630797430 & 3.8757695652 \\
\hline $\mathrm{C} 20$ & 1.6321320954 & -7.0824688946 & 2.1666067995 \\
\hline $\mathrm{C} 21$ & 2.4846411404 & -3.1577271984 & 4.4001211746 \\
\hline $\mathrm{H} 22$ & 2.7163481522 & -7.1941390959 & 2.240 \\
\hline $\mathrm{H} 23$ & 1.1762354609 & -7.3619356914 & 3.1192296627 \\
\hline $\mathrm{H} 24$ & 1.2538964781 & -7.7346505796 & 1.379 \\
\hline $\mathrm{H} 25$ & 1.9868732388 & -3.6786686963 & 471151 \\
\hline $\mathrm{H} 26$ & 3.5187921126 & -3.5102848189 & 4.3464462904 \\
\hline $\mathrm{H} 27$ & 2.4968230004 & -2.0856218787 & 4.6274394397 \\
\hline $\mathrm{H} 28$ & -0.2409715544 & -3.3793687097 & 3.819291223 \\
\hline $\mathrm{H} 29$ & 2.6366724337 & -0.8459410948 & 2.7095908632 \\
\hline C30 & 1.4000036294 & -3.5380708967 & 0.8032412742 \\
\hline H31 & 3.3680509232 & -2.9943537126 & 1.7497243625 \\
\hline H32 & 1.7927673071 & -0.7906931736 & 1.1796461588 \\
\hline C33 & 1.363713 & 212766 & -0.53 \\
\hline C34 & -1.2460198387 & -3.0519104082 & 0.9669723302 \\
\hline $\mathrm{C} 35$ & 2.7218609086 & -2.6690469205 & -1.2416184250 \\
\hline C36 & -2.5351 & 78891 & 724 \\
\hline H37 & 2.6200314853 & -2.1020249141 & -2.171846736 \\
\hline H38 & 3.4708021183 & -2.1572767310 & $-0.627235551^{\prime}$ \\
\hline H39 & 3.1235569317 & -3.6545515541 & -1.504997973 \\
\hline $\mathrm{H} 40$ & -3.3746428807 & -2.7551497339 & 1.2740178306 \\
\hline H41 & -2.7974585960 & -4.2980325214 & 1.902415119 \\
\hline $\mathrm{H} 42$ & -2.4579911847 & -2.7993189095 & 2.7807000648 \\
\hline $\mathrm{H} 43$ & 0.6377767222 & -3.2278059665 & -1.2072383750 \\
\hline H44 & 0.9778722608 & -1.7595081446 & -0.357452925 \\
\hline $\mathrm{H} 45$ & -1.0724248299 & -1.9835723550 & 0.8161088635 \\
\hline H46 & -1.3964385679 & -3.4517525996 & -0.039978332 \\
\hline $\mathrm{H} 47$ & 1.6974700251 & -4.9962068331 & 2.675024557 \\
\hline
\end{tabular}

4

SCF ENERGY at B3LYP/6-311+G** $=-763.83355$ au $\mathrm{ZPVE}=199.669 \mathrm{kcal} / \mathrm{mol}$

$\begin{array}{lccc}\text { atom } & \mathrm{x} & \mathrm{y} & \mathrm{z} \\ \text { C1 } & 0.2544541710 & -5.2052795618 & 0.6613395905 \\ \text { C2 } & -0.0503619673 & -4.7221104934 & 2.1268687006 \\ \text { C3 } & -0.6611069323 & -3.2847494361 & 2.0702558471 \\ \text { C4 } & 1.7137868419 & -3.5134940009 & 1.6654527799 \\ \text { C5 } & 0.8385614719 & -6.6532631586 & 0.4759879679 \\ \text { C6 } & 1.0246099411 & -6.9330320393 & -1.0506009109 \\ \text { C7 } & 2.2854728755 & -6.0758818341 & -1.3757368136 \\ \text { C8 } & 2.6064414808 & -5.4483120494 & 0.0193416977 \\ \text { H9 } & -1.4621066383 & -3.1792969233 & 1.3356091332 \\ \text { H10 } & 0.2713331358 & -7.4124099680 & 1.0206470727 \\ \text { H11 } & 0.1539514327 & -6.6640035774 & -1.6523123018\end{array}$




$\begin{array}{lrrr}\text { H12 } & 3.1249169566 & -6.6981629458 & -1.7004402507 \\ \text { H13 } & 2.1121366652 & -5.3346314852 & -2.1585724900 \\ \text { C14 } & 0.5954303910 & -2.4234123077 & 1.7382458367 \\ \text { N15 } & 1.3015115179 & -4.5131387832 & 2.6556309884 \\ \text { N16 } & 2.2069062987 & -6.4858498186 & 0.9757962705 \\ \text { H17 } & 3.6389981404 & -5.1154331909 & 0.1520592307 \\ \text { H18 } & -1.0823530521 & -3.0261987721 & 3.0465106329 \\ \text { C19 } & 3.0354803528 & -7.6789095701 & 1.0379461467 \\ \text { C20 } & 1.4117083972 & -4.1382897013 & 4.0557823090 \\ \text { H21 } & 4.0291881328 & -7.3980392131 & 1.4032119845 \\ \text { H22 } & 2.5985372517 & -8.3723313592 & 1.7645806782 \\ \text { H23 } & 3.1654024579 & -8.2230449347 & 0.0858170110 \\ \text { H24 } & 1.0471705049 & -4.9676768781 & 4.6714557697 \\ \text { H25 } & 2.4695134458 & -3.9866090484 & 4.2963405179 \\ \text { H26 } & 0.8623215632 & -3.2277479119 & 4.3535535367 \\ \text { H27 } & -0.6289178719 & -5.4503111581 & 2.7010291376 \\ \text { H28 } & 0.8239765800 & -1.7149894794 & 2.5402117384 \\ \text { C29 } & 1.5572462669 & -4.3164770020 & 0.3227842671 \\ \text { H30 } & 2.7309884729 & -3.1458511171 & 1.8228148537 \\ \text { H31 } & 0.4897376272 & -1.8451711022 & 0.8179226866 \\ \text { C32 } & -0.9097213755 & -5.0062610299 & -0.1985585198 \\ \text { H33 } & 1.2086168645 & -7.9999846786 & -1.2087545553 \\ \text { C34 } & 1.4454686841 & -3.4031873726 & -0.8117159554 \\ \text { N35 } & 1.4090498253 & -2.6609063893 & -1.7043919993 \\ \text { N36 } & -1.8623182382 & -4.8867461674 & -0.8524297973\end{array}$

$4 \mathrm{H}^{+}$

SCF ENERGY at B3LYP/6-311+G** $=-763.43960 \mathrm{au}$

$\mathrm{ZPVE}=208.281 \mathrm{kcal} / \mathrm{mol}$

$\begin{array}{lccc}\text { atom } & \mathrm{x} & \mathrm{y} & \mathrm{z} \\ \mathrm{C} 1 & 0.2233166616 & -5.1806262807 & 0.6333091813 \\ \mathrm{C} 2 & -0.0646372547 & -4.7294628295 & 2.1101629971 \\ \mathrm{C} 3 & -0.6405881638 & -3.2907918575 & 2.1125623469 \\ \mathrm{C} 4 & 1.7224383840 & -3.5115331478 & 1.6459749679 \\ \mathrm{C} 5 & 0.7831674897 & -6.6339874626 & 0.4695570536 \\ \text { C6 } & 1.0490964621 & -6.9614402284 & -1.0133369156 \\ \mathrm{C} 7 & 2.3108908198 & -6.1016181421 & -1.3407365030 \\ \mathrm{C} 8 & 2.6025174491 & -5.3951548370 & -0.0018765339 \\ \text { H9 } & -1.4484793226 & -3.1641412690 & 1.3888873092 \\ \text { H10 } & 0.2302283245 & -7.3838410242 & 1.0375882346 \\ \text { H11 } & 0.1874935166 & -6.7088510041 & -1.6336501627 \\ \text { H12 } & 3.1600588769 & -6.7180303336 & -1.6466342567 \\ \text { H13 } & 2.1386951844 & -5.3780437317 & -2.1393399934 \\ \text { C14 } & 0.6183941585 & -2.4324734736 & 1.7847839039 \\ \text { N15 } & 1.3312089919 & -4.5754966487 & 2.6166879815 \\ \text { N16 } & 2.1897762645 & -6.4481210047 & 1.0144541234 \\ \text { H17 } & 3.6311603439 & -5.0678995909 & 0.1574933866 \\ \text { H18 } & -1.0529481248 & -3.0539699625 & 3.0963957682 \\ \text { C19 } & 3.0468367184 & -7.6563824757 & 1.1521021893 \\ \text { C20 } & 1.4764097467 & -4.2419712265 & 4.0432113933 \\ \text { H21 } & 4.0266065467 & -7.3441993770 & 1.5190388026 \\ \text { H22 } & 2.5826482177 & -8.3274721380 & 1.8775717704 \\ \text { H23 } & 3.1583356250 & -8.1686379856 & 0.1971550369 \\ \text { H24 } & 1.1050716951 & -5.0798829806 & 4.6390325675 \\ \text { H25 } & 2.5381357668 & -4.1047617427 & 4.2650949802 \\ \text { H26 } & 0.9371929667 & -3.3355302769 & 4.3402835826 \\ \text { H27 } & -0.6397870631 & -5.4668121476 & 2.6748623230 \\ \text { H28 } & 0.8671746217 & -1.7436444023 & 2.5956917610 \\ \text { C29 } & 1.5358514676 & -4.2867258531 & 0.2931855071 \\ \text { H30 } & 2.7454455318 & -3.1588708024 & 1.7956909405 \\ \text { H31 } & 0.5065046686 & -1.8324656155 & 0.8793480694 \\ \text { C32 } & -0.9321371860 & -4.9854551985 & -0.2331677948\end{array}$




$\begin{array}{lrrr}\text { H33 } & 1.2346337302 & -8.0304100312 & -1.1459719886 \\ \text { C34 } & 1.4223212467 & -3.3812348177 & -0.8425703875 \\ \text { N35 } & 1.3671845065 & -2.6572001206 & -1.7473913980 \\ \text { N36 } & -1.8657855473 & -4.8628187325 & -0.9112090005 \\ \text { H37 } & 2.0732068849 & -5.9219661695 & 1.9436183938\end{array}$

\section{5}

SCF ENERGY at B3LYP/6-311+G** $=-576.434968 \mathrm{au}$

$\mathrm{ZPVE}=172.094 \mathrm{kcal} / \mathrm{mol}$

$\begin{array}{lccc}\text { atom } & \mathrm{x} & \mathrm{y} & \mathrm{z} \\ \mathrm{C} 1 & 0.1244538375 & -3.8241560621 & 1.6939360238 \\ \mathrm{C} 2 & 0.3307781405 & -3.1714424358 & 3.1003107681 \\ \mathrm{C} 3 & 0.4538921141 & -1.6620352517 & 2.7959772022 \\ \mathrm{C} 4 & 2.3043380751 & -2.9042594506 & 2.2199282444 \\ \mathrm{C} 5 & -0.0565517983 & -5.3724115351 & 1.5642384444 \\ \text { C6 } & -0.2397588253 & -5.6001182594 & 0.0474888132 \\ \text { C7 } & 0.9723975375 & -5.4348510471 & -0.4938848824 \\ \text { C8 } & 1.9166755516 & -5.1051640664 & 0.6830928265 \\ \text { H9 } & -0.8004555971 & -5.8209405496 & 2.2279517186 \\ \text { C10 } & 1.6662280484 & -1.4980240844 & 2.2547739094 \\ \text { N11 } & 1.7206105805 & -3.5586577617 & 3.4055345463 \\ \text { N12 } & 1.3170328408 & -5.8526682682 & 1.8039800512 \\ \text { H13 } & 2.9809959129 & -5.3087272713 & 0.5387950958 \\ \text { C14 } & 1.5006655811 & -7.2951560919 & 1.7780142485 \\ \text { C15 } & 2.2356497078 & -3.1208127292 & 4.6930160648 \\ \text { H16 } & 2.5654980273 & -7.5183521542 & 1.9102134073 \\ \text { H17 } & 0.9632729790 & -7.7352576368 & 2.6257830725 \\ \text { H18 } & 1.1548384029 & -7.7900025186 & 0.8536692259 \\ \text { H19 } & 1.6843573926 & -3.6357648306 & 5.4880374960 \\ \text { H20 } & 3.2870956382 & -3.4194268894 & 4.7732857716 \\ \text { H21 } & 2.1693825537 & -2.0335251451 & 4.8734945039 \\ \text { H22 } & -0.3863787760 & -3.4665046014 & 3.8708272351 \\ \text { C23 } & 1.5514053439 & -3.6308651629 & 1.0571970876 \\ \text { H24 } & 3.3957907140 & -2.9541497698 & 2.1835191140 \\ \text { H25 } & -0.6718724177 & -3.3066005336 & 1.1532658343 \\ \text { H26 } & 1.5520796496 & -3.0053003891 & 0.1611068551 \\ \text { H27 } & 1.2362536634 & -5.4162676530 & -1.5464931653 \\ \text { H28 } & -1.1856248086 & -5.7437146900 & -0.4650402802 \\ \text { H29 } & 2.0858612320 & -0.5972200370 & 1.8184001721 \\ \text { H30 } & -0.3360679976 & -0.9249953139 & 2.8994632450\end{array}$

\section{$\mathbf{5} \mathbf{H}^{+}$}

SCF ENERGY at B3LYP/6-311+G** $=-576.85500 \mathrm{au}$ $\mathrm{ZPVE}=181.803 \mathrm{kcal} / \mathrm{mol}$

$\begin{array}{lccc}\text { atom } & \mathrm{x} & \mathrm{y} & \mathrm{z} \\ \mathrm{C} 1 & 0.0861129434 & -3.7657036698 & 1.6341780405 \\ \mathrm{C} 2 & 0.3025246460 & -3.1674401463 & 3.0641113001 \\ \mathrm{C} 3 & 0.4833405052 & -1.6618442346 & 2.8552326459 \\ \text { C4 } & 2.2964736789 & -2.9002545312 & 2.1767407094 \\ \text { C5 } & -0.1113160764 & -5.3120066907 & 1.5371692413 \\ \text { C6 } & -0.2252580059 & -5.6599702590 & 0.0605709603 \\ \text { C7 } & 0.9871566759 & -5.4975746050 & -0.4790373657 \\ \text { C8 } & 1.9178812745 & -5.0402818444 & 0.6340356484 \\ \text { H9 } & -0.8323631119 & -5.7537560035 & 2.2262406540 \\ \text { C10 } & 1.6952177296 & -1.4994712898 & 2.3159898783 \\ \text { N11 } & 1.6980299720 & -3.6401458426 & 3.3332941977 \\ \text { N12 } & 1.3130043541 & -5.7748392635 & 1.8261003299 \\ \text { H13 } & 2.9844299149 & -5.2427204213 & 0.5274574057 \\ \text { C14 } & 1.5643462971 & -7.2329390604 & 1.9519303639 \\ \text { C15 } & 2.2449264141 & -3.3166644275 & 4.6593352505\end{array}$




$\begin{array}{lrrr}\text { H16 } & 2.6348525900 & -7.3886737220 & 2.1013027597 \\ \text { H17 } & 1.0183462485 & -7.6052432637 & 2.8212936383 \\ \text { H18 } & 1.2329426036 & -7.7508551817 & 1.0520981993 \\ \text { H19 } & 1.6783425114 & -3.8643916370 & 5.4176017911 \\ \text { H20 } & 3.2859496550 & -3.6490069227 & 4.7019901179 \\ \text { H21 } & 2.2033491149 & -2.2453842787 & 4.8883233289 \\ \text { H22 } & -0.4163848896 & -3.4867111108 & 3.8214373165 \\ \text { C23 } & 1.5211023091 & -3.5734932967 & 0.9955515136 \\ \text { H24 } & 3.3846771979 & -2.9773971801 & 2.1297916520 \\ \text { H25 } & -0.7157702795 & -3.2543198480 & 1.1017730198 \\ \text { H26 } & 1.5358750936 & -2.9527917601 & 0.0997470288 \\ \text { H27 } & 1.6242815417 & -5.2230681659 & 2.6917709603 \\ \text { H28 } & 2.1556575256 & -0.5862522693 & 1.9576700878 \\ \text { H29 } & -0.2771715386 & -0.9122942968 & 3.0401490675 \\ \text { H30 } & -1.1555651207 & -5.9068059148 & -0.4362159032 \\ \text { H31 } & 1.2775465652 & -5.5809317550 & -1.5191747383\end{array}$

\section{6}

SCF ENERGY at B3LYP/6-311+G** $=-655.07188$ au $\mathrm{ZPVE}=208.665 \mathrm{kcal} / \mathrm{mol}$

$\begin{array}{lccc}\text { atom } & \mathrm{x} & \mathrm{y} & \mathrm{z} \\ \mathrm{C} 1 & 0.0737403481 & -3.7396175071 & 1.6948774335 \\ \mathrm{C} 2 & 0.4736926045 & -3.1215844404 & 3.0958092869 \\ \mathrm{C} 3 & 0.6462952124 & -1.6038502282 & 2.8607042750 \\ \mathrm{C} 4 & 2.3475189448 & -2.9050239706 & 2.0212187825 \\ \text { C5 } & -0.0914615715 & -5.3133231130 & 1.6704220245 \\ \text { C6 } & -0.4045274712 & -5.6884381035 & 0.2041561311 \\ \text { C7 } & 0.7505681133 & -5.5550760172 & -0.4567129670 \\ \text { C8 } & 1.7834608247 & -5.0977280942 & 0.5979575520 \\ \text { H9 } & -0.7673792238 & -5.7119250810 & 2.4330464272 \\ \text { C10 } & 1.7997208835 & -1.4706968299 & 2.1969494190 \\ \text { N11 } & 1.8671926753 & -3.5693174242 & 3.2424155394 \\ \text { N12 } & 1.2994436282 & -5.7700467825 & 1.8133760504 \\ \text { H13 } & 2.8352853896 & -5.2974928033 & 0.3730913460 \\ \text { C14 } & 1.5049835431 & -7.2062191017 & 1.8819696070 \\ \text { C15 } & 2.5358064826 & -3.2086778624 & 4.4800538089 \\ \text { H16 } & 2.5814627303 & -7.4062547653 & 1.9349266937 \\ \text { H17 } & 1.0534595998 & -7.5836670385 & 2.8067146692 \\ \text { H18 } & 1.0883094014 & -7.7797158336 & 1.0348419730 \\ \text { H19 } & 2.0481268637 & -3.7324334921 & 5.3103223988 \\ \text { H20 } & 3.5741202965 & -3.5570769275 & 4.4344636510 \\ \text { H21 } & 2.5437064668 & -2.1282247094 & 4.7100954646 \\ \text { H22 } & -0.1745788197 & -3.4181335770 & 3.9259543332 \\ \text { C23 } & 1.4533561296 & -3.5811094135 & 0.9047131724 \\ \text { H24 } & 3.4256636178 & -3.0015170594 & 1.8609946153 \\ \text { C25 } & 1.4625830943 & -2.7477404744 & -0.3827702709 \\ \text { C26 } & -1.1728531082 & -3.0492840264 & 1.1269713380 \\ \text { H27 } & -1.4195880384 & -3.4124085836 & 0.1258018464 \\ \text { H28 } & -2.0412734761 & -3.2430714852 & 1.7719996985 \\ \text { H29 } & -1.0457744555 & -1.9653742422 & 1.0637857469 \\ \text { H30 } & 1.1613020608 & -1.7127298280 & -0.2010934083 \\ \text { H31 } & 2.4730793334 & -2.7269251677 & -0.8144252554 \\ \text { H32 } & 0.7904035973 & -3.1602261369 & -1.1395283154 \\ \text { H33 } & 0.9219765004 & -5.6597018899 & -1.5229718604 \\ \text { H34 } & -1.3811922374 & -5.9231025713 & -0.2060783624 \\ \text { H35 } & 2.2299710614 & -0.5624433965 & 1.7880648071 \\ \text { H36 } & -0.0709682648 & -0.8281985811 & 3.1080133754\end{array}$

$6 \mathbf{H}^{+}$ 
$\mathrm{ZPVE}=217.193 \mathrm{kcal} / \mathrm{mol}$

$\begin{array}{lccc}\text { atom } & \mathrm{x} & \mathrm{y} & \mathrm{z} \\ \mathrm{C} 1 & 0.0473872419 & -3.7052202775 & 1.6634265160 \\ \text { C2 } & 0.4498535889 & -3.1240098446 & 3.0788825095 \\ \text { C3 } & 0.6733665287 & -1.6203265462 & 2.8993005754 \\ \text { C4 } & 2.3463440531 & -2.9082451665 & 1.9941267601 \\ \text { C5 } & -0.1380357011 & -5.2735906809 & 1.6568476478 \\ \text { C6 } & -0.3902156440 & -5.7235507656 & 0.2239992106 \\ \text { C7 } & 0.7645253525 & -5.5922372651 & -0.4365379815 \\ \text { C8 } & 1.7871052088 & -5.0544663114 & 0.5554980354 \\ \text { H9 } & -0.7912414834 & -5.6723069228 & 2.4353397775 \\ \text { C10 } & 1.8276237058 & -1.4890369459 & 2.2389399816 \\ \text { N11 } & 1.8511963854 & -3.6347811745 & 3.2056013376 \\ \text { N12 } & 1.3020423289 & -5.7285175491 & 1.8285407878 \\ \text { H13 } & 2.8410313318 & -5.2588120978 & 0.3575116540 \\ \text { C14 } & 1.5663413367 & -7.1777462445 & 2.0015722534 \\ \text { C15 } & 2.5419524930 & -3.3453026008 & 4.4712893077 \\ \text { H16 } & 2.6467533968 & -7.3283129677 & 2.0570559970 \\ \text { H17 } & 1.1076676927 & -7.5039920816 & 2.9376346786 \\ \text { H18 } & 1.1537284698 & -7.7448897706 & 1.1672049988 \\ \text { H19 } & 2.0386402423 & -3.8852894573 & 5.2782619405 \\ \text { H20 } & 3.5706850774 & -3.7100265630 & 4.4021063591 \\ \text { H21 } & 2.5583694563 & -2.2768658831 & 4.7137303844 \\ \text { H22 } & -0.1993904310 & -3.4341762203 & 3.9013545301 \\ \text { C23 } & 1.4349210215 & -3.5473623923 & 0.8697843220 \\ \text { H24 } & 3.4207828163 & -3.0222143100 & 1.8305447833 \\ \text { C25 } & 1.4464213864 & -2.7151164642 & -0.4145784539 \\ \text { C26 } & -1.1962802351 & -3.0159472858 & 1.0969181410 \\ \text { H27 } & -1.4384032367 & -3.3732026949 & 0.0928781969 \\ \text { H28 } & -2.0652979448 & -3.2064574885 & 1.7389226322 \\ \text { H29 } & -1.0585402710 & -1.9340309163 & 1.0393639288 \\ \text { H30 } & 1.1457674465 & -1.6831079082 & -0.2210921547 \\ \text { H31 } & 2.4546676387 & -2.6919824943 & -0.8465819030 \\ \text { H32 } & 0.7689814110 & -3.1217677177 & -1.1695667638 \\ \text { H33 } & 0.9588107293 & -5.7609627917 & -1.4883409397 \\ \text { H34 } & -1.3552215688 & -6.0238620550 & -0.1647412501 \\ \text { H35 } & 2.2946685841 & -0.5802376882 & 1.8788086481 \\ \text { H36 } & -0.0181855972 & -0.8433371686 & 3.2023098661 \\ \text { H37 } & 1.6973741934 & -5.1241298811 & 2.6400344377\end{array}$

7

SCF ENERGY at B3LYP/6-311+G** $=-760.95271 \mathrm{au}$ $\mathrm{ZPVE}=170.189 \mathrm{kcal} / \mathrm{mol}$

$\begin{array}{lccc}\text { atom } & \mathrm{x} & \mathrm{y} & \mathrm{z} \\ \mathrm{C} 1 & 0.2668101319 & -5.2106202826 & 0.6737756593 \\ \text { C2 } & -0.0475766662 & -4.7055745867 & 2.1424648508 \\ \text { C3 } & -0.5629186648 & -3.2622195457 & 1.9671809902 \\ \text { C4 } & 1.7056929319 & -3.5066559655 & 1.6854470658 \\ \text { C5 } & 0.8549694259 & -6.6675760061 & 0.4673076457 \\ \text { C6 } & 1.0679966146 & -6.8011275428 & -1.0547201944 \\ \text { C7 } & 2.1474613244 & -6.0634810124 & -1.3352813506 \\ \text { C8 } & 2.6097560665 & -5.4700378401 & 0.0114230592 \\ \text { H9 } & 0.2799998627 & -7.4504974452 & 0.9658700166 \\ \text { C10 } & 0.5160179715 & -2.5245935491 & 1.6846574040 \\ \text { N11 } & 1.3071580599 & -4.5161295761 & 2.6721155268 \\ \text { N12 } & 2.2175512269 & -6.4966179182 & 0.9830499033 \\ \text { H13 } & 3.6506420342 & -5.1498962266 & 0.0899196156 \\ \text { C14 } & 3.0432591801 & -7.6956368056 & 1.0125320207 \\ \text { C15 } & 1.3948844989 & -4.1130319216 & 4.0685842204 \\ \text { H16 } & 4.0439902548 & -7.4231416808 & 1.3636324429\end{array}$




$\begin{array}{lrrr}\text { H17 } & 2.6158571226 & -8.3976731215 & 1.7361036302 \\ \text { H18 } & 3.1404323030 & -8.2090877481 & 0.0416007281 \\ \text { H19 } & 1.0219718472 & -4.9312525227 & 4.6935181289 \\ \text { H20 } & 2.4482581598 & -3.9538039349 & 4.3216229306 \\ \text { H21 } & 0.8326015951 & -3.1978121683 & 4.3175986548 \\ \text { H22 } & -0.6646221130 & -5.3939026581 & 2.7228211926 \\ \text { C23 } & 1.5618363085 & -4.3266452341 & 0.3369449281 \\ \text { H24 } & 2.7023297858 & -3.0901534131 & 1.8445674790 \\ \text { C25 } & -0.9040081038 & -5.0196107761 & -0.1811050296 \\ \text { C26 } & 1.4589755787 & -3.4081698737 & -0.7956377318 \\ \text { N27 } & 1.4512903691 & -2.6564928860 & -1.6809278154 \\ \text { N28 } & -1.8694237297 & -4.9205882945 & -0.8191696581 \\ \text { H29 } & -1.6025513455 & -2.9569697310 & 1.9612641042 \\ \text { H30 } & 0.5563985308 & -1.4802850874 & 1.3986191045 \\ \text { H31 } & 0.3988316989 & -7.2979590107 & -1.7471265473 \\ \text { H32 } & 2.5598733237 & -5.8241944654 & -2.3082478683\end{array}$

\section{$\mathbf{7} \mathbf{H}^{+}$}

SCF ENERGY at B3LYP/6-311+G** $=-761.34278 \mathrm{au}$ $\mathrm{ZPVE}=179.500 \mathrm{kcal} / \mathrm{mol}$

$\begin{array}{lccc}\text { atom } & \mathrm{x} & \mathrm{y} & \mathrm{z} \\ \text { C1 } & 0.2378980204 & -5.1974572137 & 0.6254081026 \\ \text { C2 } & -0.0890159323 & -4.7220420014 & 2.0963946413 \\ \text { C3 } & -0.5429731099 & -3.2714407820 & 2.0220647199 \\ \text { C4 } & 1.7150842487 & -3.4904601751 & 1.6290402476 \\ \text { C5 } & 0.8327205267 & -6.6571273046 & 0.4680768835 \\ \text { C6 } & 1.1077525720 & -6.8450572891 & -1.0247291181 \\ \text { C7 } & 2.1871885653 & -6.1091190622 & -1.3033791605 \\ \text { C8 } & 2.6074308009 & -5.4473642427 & 0.0099521923 \\ \text { H9 } & 0.2534327595 & -7.4276482766 & 0.9789544087 \\ \text { C10 } & 0.5362080886 & -2.5347787960 & 1.7420694357 \\ \text { N11 } & 1.3199324041 & -4.5547333849 & 2.6374532172 \\ \text { N12 } & 2.1917910815 & -6.4394589134 & 1.0433432862 \\ \text { H13 } & 3.6429718231 & -5.1167960281 & 0.1036026338 \\ \text { C14 } & 3.0402586574 & -7.6393304008 & 1.1601859549 \\ \text { C15 } & 1.4745862498 & -4.2317428215 & 4.0825806071 \\ \text { H16 } & 4.0255872097 & -7.3364326720 & 1.5236321527 \\ \text { H17 } & 2.5925180505 & -8.3138745252 & 1.8944901672 \\ \text { H18 } & 3.1559082538 & -8.1696948657 & 0.2088580004 \\ \text { H19 } & 1.0978385723 & -5.0750340514 & 4.6646183996 \\ \text { H20 } & 2.5369905007 & -4.0898319824 & 4.2901177218 \\ \text { H21 } & 0.9192350862 & -3.3270515281 & 4.3278755540 \\ \text { H22 } & -0.6845882204 & -5.4229070337 & 2.6820459592 \\ \text { C23 } & 1.5428279624 & -4.3079330133 & 0.2880850413 \\ \text { H24 } & 2.7183816337 & -3.0994338747 & 1.8005431711 \\ \text { C25 } & -0.9305262511 & -5.0001809206 & -0.2238054819 \\ \text { C26 } & 1.4402626572 & -3.3861548784 & -0.8363659773 \\ \text { N27 } & 1.4119103207 & -2.6295257493 & -1.7154780020 \\ \text { N28 } & -1.8900573722 & -4.8754157685 & -0.8640739553 \\ \text { H29 } & -1.5755575259 & -2.9549497266 & 2.0965620843 \\ \text { H30 } & 0.5934527918 & -1.4739262728 & 1.5341635495 \\ \text { H31 } & 0.4746923786 & -7.3995247326 & -1.7063032179 \\ \text { H32 } & 2.6451484965 & -5.9208737981 & -2.2664603688 \\ \text { H33 } & 1.8525334480 & -5.4442580175 & 2.3518298035 \\ & & & \end{array}$




\section{Isodemic setup for the bases 1 and 2 :}

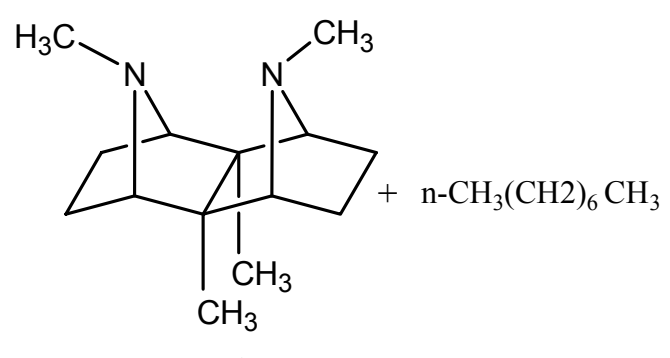

2

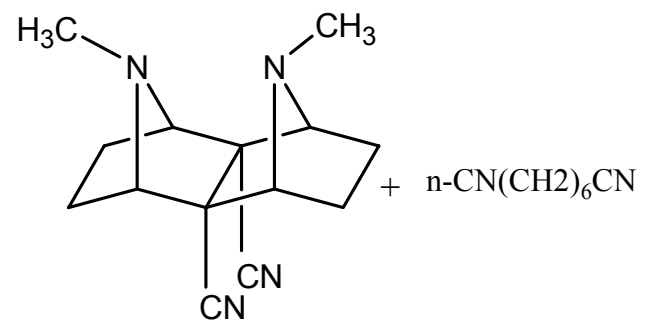

4
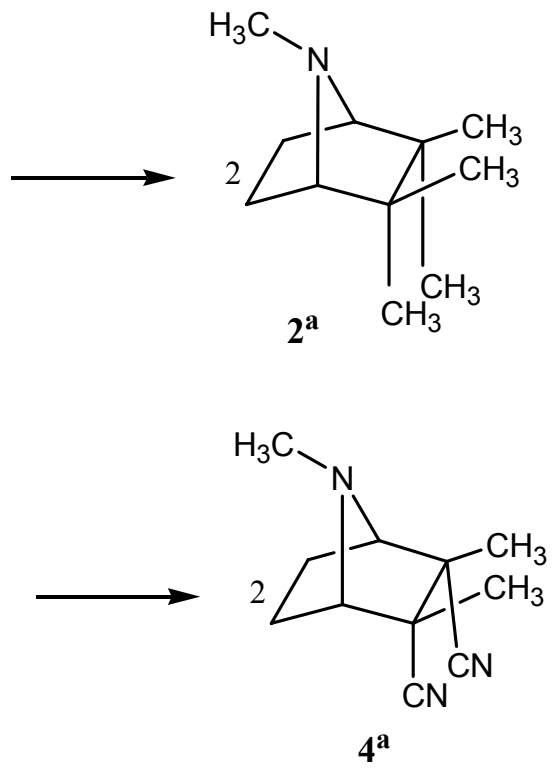

$4^{\mathrm{a}}$

The strain energy (SE) of the unprotonated proton sponge is given by:

$\mathrm{SE}(2)=\mathrm{E}(2)+\mathrm{E}\left(\mathrm{n}-\mathrm{CH}_{3}\left(\mathrm{CH}_{2}\right)_{6} \mathrm{CH}_{3}\right)-2 \mathrm{E}\left(2^{\mathrm{a}}\right)$

The convention chosen ensure that the strain energy will be a positive quantity (destabilizing). 